\title{
Localización óptima de un relleno sanitario empleando sistemas de información geográfica distrito de Chachapoyas Amazonas- 2017
}

\author{
Optimal location of a sanitary landfill using geographic information systems \\ Chachapoyas district Amazonas- 2017
}

\author{
Angers William Espejo Pingus
}

\section{RESUMEN}

La presente investigación surge como una alternativa a los problemas socio ambientales que se generaron en el proyecto SNIP - 146148 "Mejoramiento y ampliación de la gestión integral de los residuos sólidos municipales del distrito de Chachapoyas, provincia de Chachapoyas, región Amazonas ", específicamente los impactos del estudio de selección de sitio para la disposición final de residuos sólidos en la ciudad de Chachapoyas, sobre el suelo, aire, agua y la vulneración de los derechos fundamentales. El objetivo fue localizar una área óptima (técnica y ambientalmente adecuada) para un relleno sanitario empleando un sistema de información geográfica - SIG, integrado a los criterios de selección de sitio como pendiente, geología, distancia a carreteras, hidrología, bosques, distancia a la población urbana - rural, distancia a un aeropuerto y el volumen de almacenamiento, estos criterios se evaluaron mediante la evaluación multicriterio y los pesos para cada criterio fueron de 0 para lugares no óptimos y 1 para lugares óptimos, empleando los SIG se obtuvieron cuatro zonas óptimas dentro del área de estudio, cada uno de ellas con sus áreas correspondientes: Área $01=60.43$ Has., Área $02=6.91$ Has., Área $03=3.1$ Has., y Área $04=$ 15.1 Has. La metodología utilizada tuvo una fase de campo, en la cual la recopilación de información y la toma de puntos de control con GPS fue in situ, y en la fase de gabinete se determinaron la caracterización de la gestión de los residuos sólidos, los criterios de selección de sitio, la elaboración de los mapas temáticos, la superposición de mapas y posteriormente el análisis e interpretación. El distrito de Chachapoyas cuenta con una población de 23399 habitantes según la tasa de crecimiento de la población. Mientras que se estima que en el año 2025 la población sea de 30275 habitantes, a medida que la población y la urbanización aumenta, el consumo aumenta y con ello los residuos sólidos es un gran problema para la gestión municipal, por lo tanto, elegir el sitio óptimo para un relleno sanitario es muy crucial.

Palabras clave: Relleno sanitario, gas invernadero, cambio climático, SIG aplicado a la gestión ambiental.

\begin{abstract}
The present research arises to solve the social and environmental problems generated by the project SNIP - 146148 "Improvement and extension of the integral management of municipal solid waste in the district of Chachapoyas, province Chachapoyas, Amazonas region" specifically the impacts of the site selection study for the final disposal site of solid waste for the city of Chachapoyas, on soil, air, water and violation of fundamental rights. The objective was to locate an optimal area (technically and environmentally adequate) for a landfill using a geographic information system (GIS), the use of GIS integrated with site selection criteria such as slope, geology, distance to roads, hydrology, forests, distance to the urban - rural population, distance to an airport and the volume of storage, these criteria were evaluated by the multicriteria evaluation and the weights for each criterion were 0 for non optimal places and 1 for optimal places, using GIS four optimal areas were obtained within the study area, each with its corresponding areas: Area $01=60.43 \mathrm{Ha}$., Area $02=6.91 \mathrm{Ha}$., Area $03=3.1 \mathrm{Ha}$., and Area $04=15.1 \mathrm{Ha}$. The methodology used was: in the field phase, the collection of information in situ and the acquisition of control points with GPS, in the cabinet phase, the characterization of the solid waste management, site selection criteria, the elaboration of the thematic maps, the superposition of maps and later the analysis and interpretation. The district of Chachapoyas has a population of 23399 inhabitants according to the rate of population growth in the year 2025 the population will be 30275 inhabitants, as population and urbanization increases, consumption increases and thus solid waste in a big problem for the management municipal, therefore choosing the optimal site for a landfill is very crucial.
\end{abstract}

Keywords: Landfill, greenhouse gas, climate, change, GIS applied to environmental management. 


\section{INTRODUCCIÓN}

La población estimada en el año 2017 es de 7.5 mil millones de personas (Genial, 2017), para el año 2100 la población mundial será de 11 mil millones de personas (UNIDAS, 2015), a medida que la población, la urbanización y el consumo aumentan, los residuos sólidos se hacen un mayor problema (Mihelcic y Zimmerman, 2012), los residuos sólidos son dispuestos en lugares sin ningún tipo de medida técnica y ambiental (Castañeda, Montoya, y Mejía, 2010), la producción per cápita mundial de residuos sólidos en países industrializados es de 1 kilogramo por persona (Mihelcic y Zimmerman, 2012), en Perú la producción per cápita va desde 0.47 - 0.65 kilogramos por habitante (OEFA, 2015).

El aire, el agua y el suelo, constituyen los medios donde se vierten los residuos generados por el hombre (Mena et al., 2010). El manejo inadecuado de los residuos sólidos representa un peligro para la salud de las personas, el riesgo es mayor si los residuos son dispuestos en botaderos (MINAM, 2011b), en el Perú el $83 \%$ de la producción total de los residuos sólidos es destinado a lugares inadecuados, causando daños al ambiente y salud de las personas (MINAM, 2011b), el principal problema del manejo de residuos sólidos es la escasez de lugares adecuados destinados a su disposición (MINAM, 2016).

Una herramienta común para evaluar los sitios potenciales de rellenos sanitarios es un sistema de información geográfica (GIS). Este sistema, es un medio valioso para el procesamiento de grandes cantidades de datos y para asegurar una evaluación de todas las opciones (Mihelcic y Zimmerman, 2012), además logra sintetizar las variables que condicionan la construcción y operación de rellenos sanitarios, teniendo en cuenta las características físicas, hidrológicas, socioculturales y técnicas que permiten seleccionar las mejores opciones, mediante los SIG se puede recolectar, manipular, interactuar y analizar la información final (Castañeda et al., 2010), el GIS también permite al ingeniero optimizar los diagnósticos ambientales y tomar mejores decisiones geo ambientales (Mihelcic y Zimmerman, 2012).

\section{MATERIAL Y MÉTODO}

\section{a. Ubicación}

La investigación se realizó en el distrito y provincia de Chachapoyas, región Amazonas, Perú, Geográficamente se encuentra entre las coordenadas UTM 182118.80 E y 9310572.94 S, ocupando una superficie de $153.40 \mathrm{~km} 2$. De acuerdo con SIGERSOL 2015 el distrito de Chachapoyas cuenta con una población de 23399 habitantes, de estos 23221 hab. Es población urbana y 178 hab. pertenecientes a población rural, con una densidad de 155,67 hab./km2 (MPCH Chachapoyas, 2013).

\section{b. Insumos o datos cartográficos}

Los insumos cartográficos vienen con información técnica y básica para el manejo e interpretación de los productos cartográficos, descargadas de geoservidores gratuitos de carácter oficial tanto nacional e internacional (Instituto Geográfico Nacional, Ministerio del Ambiente, GEOCATMIN, Transportes y comunicaciones, Servicio Nacional de Meteorología e Hidrología) e imágenes satelitales de Google Eatrh y de servidores gratuitos como GLCF (Global Land Cover Facility).

\section{a. Métodos, técnicas e instrumentos de recolección de datos}

La investigación se desarrolló en tres fases que se detallan a continuación:

Fase pre-campo:

Se revisó investigaciones sobre el tema de estudio tanto de alcance nacional e internacional, se recolecto información e insumos cartográficos de los geo-servidores nacionales y plataformas virtuales, posteriormente se diseñó el plan de trabajo de la investigación, a continuación, se identificaron y determinaron los criterios de selección de sitio

Fase de gabinete - campo:

- Caracterización de gestión integral de los residuos sólidos del distrito y provincia de Chachapoyas.

En esta etapa se determinó la generación y composición de residuos sólidos municipales del distrito de Chachapoyas, densidad de los residuos sólidos, la proyección de la producción per cápita de residuos sólidos, se calculó del área requerida para el relleno sanitario del distrito de Chachapoyas.

- Criterios y factores óptimos de localización de un relleno sanitario en el distrito de Chachapoyas región Amazonas. Durante esta etapa se logró determinar los criterios mínimos para la localización óptima del relleno sanitario en el distrito de Chachapoyas, estos criterios permitieron determinar la ubicación más conveniente para un relleno sanitario. 
Tabla 1. Criterios de selección utilizados y su valor en software GIS

\begin{tabular}{|c|c|}
\hline CRITERIO & ESCALA \\
\hline Geología & $\begin{array}{c}\text { Estratigrafía } \\
\text { Precambrico } \\
\text { Paleozoico o Mesozoico }\end{array}$ \\
\hline Hidrografía & Distancia $>500 \mathrm{~m}$ \\
\hline Pendiente & 4 a 40 grados \\
\hline Cobertura Vegetal & $\begin{array}{c}\text { Herbazales o zonas } \\
\text { degradadas }\end{array}$ \\
\hline Aeropuerto & Distancia $>2000 \mathrm{~m}$ \\
\hline Vías de Acceso & Distancia $250>2000 \mathrm{~m}$ \\
\hline Zona urbana & Distancia $>2000 \mathrm{~m}$ \\
\hline Correferenciación & Error aceptable $>0-5 \mathrm{~m}$ \\
\hline Ponderación & $\begin{array}{c}\text { Valores de 0-1 (1:apto, } \\
0: \text { No apto) }\end{array}$ \\
\hline $\begin{array}{c}\text { Información } \\
\text { vectorial }\end{array}$ & $<3$ metros de error \\
\hline Puntos de muestreo & $\begin{array}{c}\text { Coordenadas } \\
\text { Geográficas }\end{array}$ \\
\hline
\end{tabular}

Fuente: (Silva, 2015), (CAEG, 2017) y (MINAM, 2011)

\section{- Criterios desde el punto de vista técnica profesional y social}

1.Criterio de pendiente. (MINAM, 2011b), (Celestino Ordóñez Galán, 2003) el vertedero deberá ubicarse en áreas con pendientes inferiores a $40^{\circ}$ con el objeto de evitar las posibles problemáticas de inestabilidad, la pendiente inferior sea superior a 40 , de forma que no se originen encharcamientos en el entorno del área de vertido.

2. Criterio geológico. Dada la necesidad de que el sustrato de la instalación de vertido sea impermeable, para evitar la contaminación de acuíferos subterráneos, y a fin de reducir costesde impermeabilización artificial, se adopta como criterio geológico que el relleno sanitario esté ubicado sobre un sustrato arcilloso (Ordóñez, 2003), (Gómez et al., 2005).

3.Criterio de distancia a carreteras. A fin de reducir los costes de transporte y mantenimiento de los vehículos, se plantea que la instalación de vertido deberá estar próxima a una carretera de primer y segundo orden, pero evitando una excesiva proximidad, a fin de evitar, en lo posible, su intervisibilidad. Por ello, el criterio empleado es que la distancia a una carretera debe estar comprendida entre 250 y $2.000 \mathrm{~m}$.Vías de acceso (Ordóñez, 2003) y (MINAM, 2011).

4. Criterio hidrológico. Con objeto de reducir el riesgo de potenciales contaminaciones directas e indirectas a cauces fluviales, la instalación de vertido se ubicó a una distancia no inferior a $500 \mathrm{~m}$ de cualquier cauce fluvial permanente (Gómez et al., 2005), el sitio seleccionado esté lo más lejos posible de corrientes superficiales y cuerpos receptores de agua, y cuente con una adecuada red de drenaje pluvial para evitar escurrimientos dentro del relleno sanitario. (MINAM, 2011)

5. Criterio de usos del suelo forestales. Los condicionantes planteados, desde el punto de vista de usos del suelo forestales, han sido los de evitar aquellas zonas de especies forestales con un marcado interés ecológico, (Ordoñez, 2003).

6. Criterio de distancias a núcleos urbanos. Dado que este tipo de instalaciones genera un cierto rechazo social por efecto de la producción de olores, así como por los posibles riesgos que pueda suponer, se planteó como condición de ubicación que esté situado a una distancia no inferior a 2.000 $\mathrm{m}$ de cualquier núcleo urbano. Distancia $>3000 \mathrm{~m}$ de aeropuertos (MINAM, 2011).

7.Criterio de volumen de almacenamiento. Puesto que una instalación de vertido de RSU significa una inversión importante, es imprescindible que tenga una capacidad de almacenamiento suficiente para el período proyectado.

\section{- Recolección y generación de datos cartográficos}

Se trabajó con datos cartográficos de carácter oficial tanto nacional como internacional, además se recolecto información de variables topográficas, naturales, territoriales, antrópicas, entre otras, las cuales fueron adquiridas mediante la descarga de información, recolección de datos mediante GPS o en otros casos mediante procesos para generar nueva información.

\section{Fase de gabinete}

-Homogenidad de datos cartográficos

En la presente investigación se evaluó variables cuantitativas, se analizó mediante archivos raster, por ello, todolos datos empleadas en nuestro análisis, fueron rasterizadas bajo un mismo tamaño de cuadricula o tamaño de pixel.

-Elaboración de mapas temáticos de cada criterio 
óptimo de selección de sitio.

Para la elaboración de los mapas de los criterios de selección de sitio se usó el software ArcGis v. 10.3 y Google Earth.
- Superposición de criterios

Una vez que se construyó todo los mapas de restricciones de cada criterio de selección de sitio, se procedió a combinarlos mediante una intersección para obtener las zonas que cumplen todas y cada una de las condiciones establecidas.

\begin{tabular}{|c|c|c|}
\hline \multicolumn{3}{|c|}{ FASE DE PRE CAMPO: RECOLECCIÓN DE INFORMACIÓN } \\
\hline $\begin{array}{l}\text { Identificaron los Criterios } \\
\text { de selección de sitios }\end{array}$ & $\begin{array}{l}\text { Mapas temáticos e insumos } \\
\text { cartográficos }\end{array}$ & $\begin{array}{l}\text { Diagnóstico - caracterización } \\
\text { integral de R.R.S.S. }\end{array}$ \\
\hline \multicolumn{3}{|c|}{$\begin{array}{l}\text { FASE DE GABINETE - CAMPO : EVALUACION DE LA CALIDAD DE } \\
\text { SUELO }\end{array}$} \\
\hline $\begin{array}{l}\text { Integración de mapas } \\
\text { temáticos }\end{array}$ & $\begin{array}{l}\text { Evaluación de mapas y } \\
\text { resultados del proceso }\end{array}$ & $\begin{array}{l}\text { Evaluar las opciones } \\
\text { preseleccionadas con criterios } \\
\text { de selección de sitio en campo }\end{array}$ \\
\hline \multicolumn{3}{|c|}{$\begin{array}{l}\text { FASE DE GABINETE: ANÁLISIS DE DATOS. ELABORACIÓN FINAL DE } \\
\text { INFORME DE INVESTIGACIÓN }\end{array}$} \\
\hline $\begin{array}{l}\text { Procesamiento de } \\
\text { información }\end{array}$ & Análisis Estadístico & $\begin{array}{c}\text { Generación final del informe } \\
\text { de investigación }\end{array}$ \\
\hline
\end{tabular}

Figura 1: Fases metodológicas desarrolladas

\section{RESULTADOS}

3.1. Caracterización integral de los residuos sólidos. Chachapoyas cuenta con una población de 23339 habitantes, la generación per cápita de residuos sólidos fue de $0.47 \mathrm{~kg} / \mathrm{hab}$./día, y, se generó 10.97 t/día y 95.55 toneladas / semana. En Chachapoyas el $62.28 \%$ de los residuos sólidos generados fueron orgánicos y el $37.71 \%$ fueron residuos sólidosinorgánicos.

Para el año 2025 la población en el distrito de Chachapoyas será de 30275 habitantes, la producción per cápita será de $0.52 \mathrm{~kg} / \mathrm{hab}$./día y el área mínima requerida para el relleno sanitario en el distrito de Chachapoyas fue de 30180.95 $\mathrm{m} 2$ o 3.02 hectáreas.

\subsection{Análisis multicriterio}

En el criterio pendiente, el relleno sanitario está sobre áreas superiores a $4^{\circ} \mathrm{y}$ en áreasinferiores a $40^{\circ}$, para facilitar la construcción del relleno sanitario y no generar encharcamiento dentro del área del relleno sanitario, en el criterio geología, el sustrato de la instalación del relleno sanitario es impermeable, para evitar la contaminación de acuíferos subterráneos, el relleno sanitario se ubica sobre sustrato arcilloso.

Criterio de distancia a carreteras, a fin de reducir los costos detransporte y mantenimiento, el relleno sanitario está ubicado próximo a una carreta, la distancia próxima de una carretera comprende entre 250 y $2000 \mathrm{~m}$. en segundo término el criterio hidrológico, el relleno sanitario está ubicado a una distancia no inferior a $500 \mathrm{~m}$ de cualquier cauce fluvial, con el objeto de reducir el riesgo de contaminación a cauces fluviales.

Para el criterio de uso de suelos forestales, las áreas del relleno sanitario evitan aquellas zonas con especies forestales, bosques primarios o zonas marcadas como interés ecológico y para el criterio de distancia a núcleos urbanos - rurales, los rellenos sanitarios están ubicados a una distancia no inferior a $2000 \mathrm{~m}$ de cualquier núcleo urbano y rural con la finalidad que genere aceptación social, malos olores, riesgos a sus derechos fundamentales. También se consideró la misma distancia para el aeropuerto.

Criterio de volumen de almacenamiento, el proyecto de relleno sanitario tiene una vida útil de 10 años, los rellenos sanitarios cuentan con un área mínima de 3 hectáreas y como máxima 70 hectáreas, necesarias para el periodo proyectado.

Sistemas de información geográfica aplicada a la gestión ambiental:

Mediante el empleo de los Sistemas de 
Información Geográfica (SIG) y la superposición de los criterios de localización óptima de relleno sanitario para el distrito de Chachapoyas, se obtuvieron como resultado cuatro áreas definidas como zonas Óptimas, las más adecuadas para el establecimiento de las instalaciones para el relleno sanitario. Área $01=60.43 \mathrm{Ha}$., Área $02=$ 6.91 Ha., Área 03 = 3.1Ha., y Área $04=15.1 \mathrm{Ha}$; ubicadas en el Distrito de Chachapoyas.

Se elaboraron un total de 7 mapas temáticos de los criterios óptimos para la localización óptima de un relleno sanitario en el distrito de
Chachapoyas divididos en estudios del medio: biológico (1), físico (3) y social (3).

Se realizó la descripción de las zonas óptimas para un relleno sanitario en el distrito de Chachapoyas como clima, vulnerabilidad, uso actual del suelo, zonas de vida.

Se elaboró una comparación entre las zonas óptimas del relleno sanitario vs el tiradero actual de Rondón, el tiradero de Rondón no cumple con más del $50 \%$ de los criterios de localización óptimas para un relleno sanitario en el distrito de Chachapoyas.

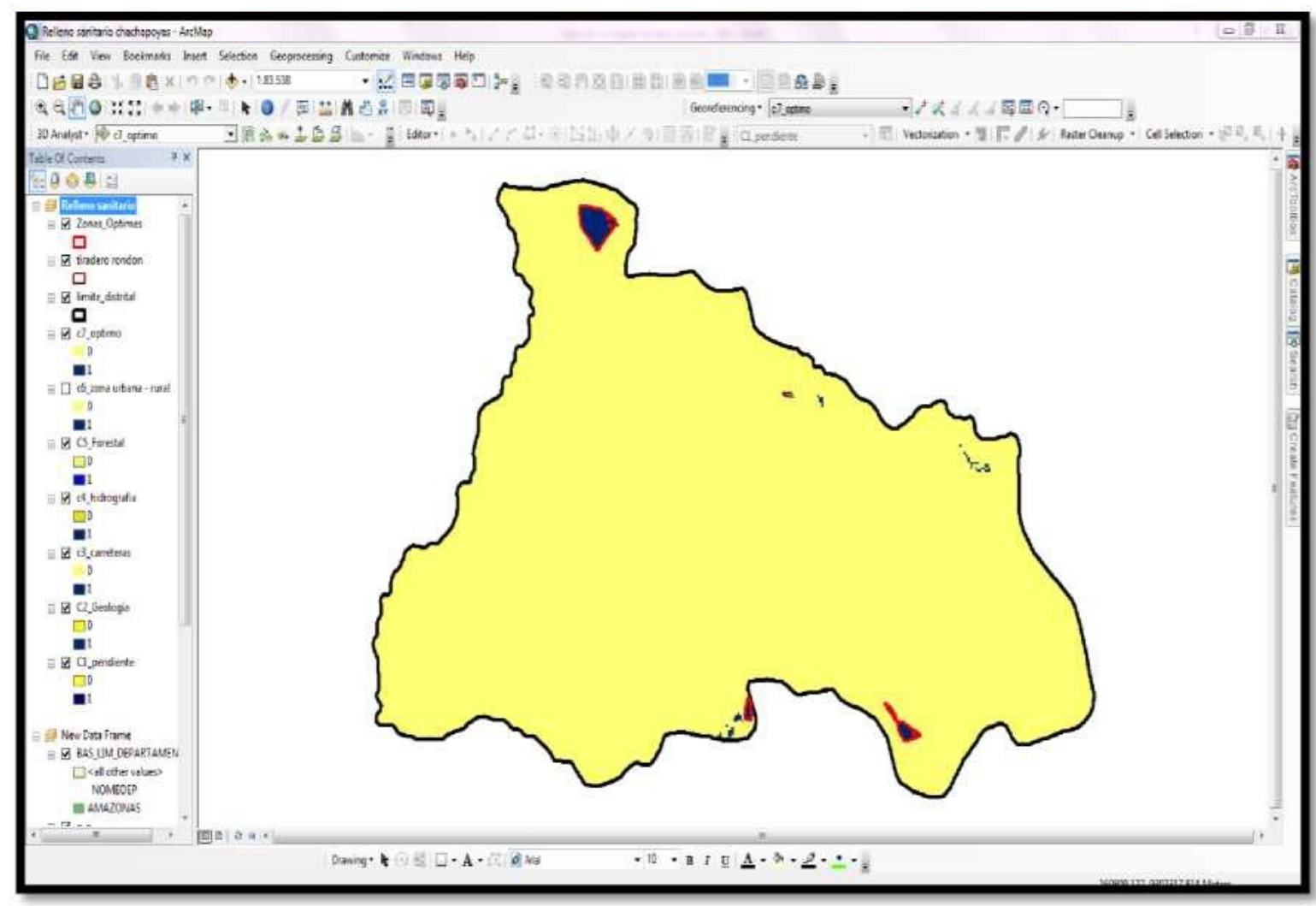

Figura 2: Sistemas de información geográfica aplicado a la gestión ambiental

\section{DISCUSIÓN}

(Mihelcic y Zimmerman, 2012) mencionan que las fuentes de desperdicio de residuos sólidos provienen de las viviendas, que comprenden los desperdicios de alimentos, desperdicios de patio o jardín, papel, plástico, vidrio, metal y el porcentaje típico generados comprenden entre el $30 \%$ y $50 \%$ del desperdicio solido municipal, en Chachapoyas el $62.28 \%$ de los residuos sólidos generados son orgánicos y el $37.71 \%$ son residuos sólidos inorgánicos; La generación per cápita de residuos sólidos en países industrializados para el año 2005 fue 0.75 a $1.5 \mathrm{mg}$ por persona por año y los desperdicio sólido generado en los países en desarrollo es de 0.15 a $0.3 \mathrm{mg}$ por persona por año, la composición del desperdicio también es diferente en los países en desarrollo generan más residuos orgánicos y la menorfracción de productos manufacturados como papel, metales y vidrio, en la presente investigación la generación per cápita es de $1.2 \mathrm{mg}$ por persona por año en el 2015 y en el 2025 será de $1.4 \mathrm{mg}$ por persona por año.

(Sosa y Torres, 2010) determinaron que aplicando el SIG y criterios de selección de sitio para la construcción de un relleno sanitario, los SIG indicaron ocho sitios con áreas mayores de seis hectáreas que cumplieron con todos los criterios normados y técnicos en la presente investigación los 
SIG indicaron cuatro áreas mayores de tres hectáreas y menores a 70 hectáreas que cumplen con todo los criterios técnicos, ambientales y sociales, (Mihelcic y Zimmerman, 2012) indicaron que los emplazamiento de rellenos sanitarios necesitan estar ubicados en donde los riesgos al medio ambiente y la sociedad son bajos, por lo que aun en el caso de un mal diseño, construcción u operación, el riesgo resultante es minimizado. (Silva, 2015) determinó 19 criterios biológico (3), físico (9) y socioeconómico (7), (MINAM, 2011) y (Mihelcic y Zimmerman, 2012) establecieron algunos criterios de selección de sitio para un relleno sanitario, el relleno sanitario debe evitar llanuras, fallas geológicas, pendientes muy pronunciadas, geología, áreas con ecosistemas significativos y biodiversidad importante, áreas de relevancia cultural o arqueológica, cuencas de agua, minimizar los costos de transportación, material para cobertura y la aceptación social donde no se perjudique los derechos fundamentales, los criterios de selección de sitio propuestos en la presente investigación tiene como objetivo proteger la salud pública, proteger el medio ambiente y la diversidad biológica, minimizar conflictos sociales y minimizar el costo para la municipalidad de Chachapoyas, se determinaron un total de 7 criterios de selección de sitio para un relleno sanitario.

\section{CONCLUSIONES}

Se elaboró una metodología de geo-procesos empleando sistemas de información geográfica que permiten el análisis del territorio determinando la localización técnica y ambientalmente adecuada para las instalaciones de rellenos sanitarios cuyos resultados fueron cuatro áreas óptimas.

- El empleo y aplicación de los sistemas de información geográfica permitieron el procesamiento datos raster y permite al profesional utilizarlo como un instrumento para el análisis de riesgos, minimizar las problemáticas medioambientales y tomar mejor decisiones geoambientales para asegurar la viabilidad de un proyecto. Esta investigación es uno de los ejemplos prácticos de selección de sitios para el emplazamiento de relleno sanitario empleando los SIG. La metodología es simple y eficiente para resolver este tipo de problemas, este enfoque se puede ampliar en todo el departamento de Amazonas.

-Se determinó cuatro áreas óptimas donde es posible ubicar un relleno sanitario en el distrito de Chachapoyas, los lugares se encuentran por las siguientes coordenadas (Área 1: 180579.63 m E y
$9315438.18 \mathrm{~m} \mathrm{~S}$, área 2: $184807.43 \mathrm{~m} \mathrm{E} \mathrm{y}$ $9304801.58 \mathrm{~m} \mathrm{~S}$, área 3: $188815.19 \mathrm{~m} \mathrm{E} \mathrm{y}$ $9304738.20 \mathrm{~m} \mathrm{~S}$ y área 4: $189203.29 \mathrm{~m} \mathrm{E} \mathrm{y}$ $9304321.32 \mathrm{~m} \mathrm{~S})$ que cumple con todos los requerimientos ambientales, sociales.

- Se determinaron siete criterios y factores óptimos de localización para un relleno sanitario.

- Los resultados obtenidos en este estudio, demuestra que empleando los Sistemas de Información Geográfica (SIG) y sus diferentes herramientas, determinan las zonas óptimas para ubicar el relleno sanitario para el distrito de Chachapoyas que ocasiones un menor impacto ambiental.

\section{REFERENCIAS BIBLIOGRÁFICAS}

Aksoy, E., \& San, B. (2016). Using MCDA and GIS for landfill site selection: Central districts of antalya province. International Archives of the Photogrammetry, Remóte Sensing and Spatial Information Sciences - ISPRS Archives, 41 (July), 151-157. https://dpi.org/10.5194/isprsarchives XLI-B2-151-2016

Becerra, C., Castro M. y Rodríguez, A. (2015). Identificación de áreas potenciales para la disposición final de residuos sólidos del municipio de Popayán, 82.

CAEG. (2017). AMC Para la ubicacion de un relleno de residuos sólidos. Lima.

Castañeda,F., Montoya, P. y M e j í a , Z.(2010). Metodología para la localización de rellenos sanitarios mediante $\mathrm{s}$ i s t e $\mathrm{m} \mathrm{a} \mathrm{s} \quad \mathrm{d} \mathrm{e}$ información geográfica. Un caso regional colombiano.

Castro, W. (2007). Zonificación Ecológica y Económica del departamento de Amazonas - Chachapoyas.

Ordóñez Galán C. (2003). Sistemas de Información Geográfica: Aplicaciones Prácticas con Idrisi32 al análisis de riesgos naturales y problemáticas medioambientales. ( $\mathrm{R}$. Ma, Ed.) (Primera ed). Madrid.

Congreso de la República. (2000). Ley General de Residuos Sólidos.

Fernández, S., y Del Río, J. (2011). Sistemas de Información Geográfica para el ordenamiento territorial. Serie Documentos de Gestión Urbana.

Genial. (2017). Crecimiento de la poblacion mundial. Retrieved fro m https://web.facebook.com/GenialGuru/vi deos/1990363101284828

Gómez, Montserrat y Barredo, J. (2005). Sistemas de 
Información Geográfica y evaluación multicriterio en la ordenación del territorio. (segunda edición). España.

Instituto Superior del Medio Ambiente. (n.d.). Manual de Formación del Curso Práctico Técnico GIS Aplicado a la Gestión Ambiental ARCGIS

ISO. (2005). ISO - 19128. Geographic information - Web map server interface. Switzerland.

Liliana, S. (2010). Introduccion a la Ingenieria Ambiental para la Industria de Procesos. $\mathrm{R}$ e $\mathrm{t} \mathrm{r}$ i e $\mathrm{v}$ e d $\mathrm{f} \mathrm{r}$ o $\mathrm{m}$ http;//dspace.universia.net/handle/2024/ 594

Mena, Carlos, Morales Hernández, Yohana, Ormazábal Rojas, Yony, Gajardo Valenzuela, J. (2010). Localización de un relleno sanitario en la comuna de parral, chile, a través de evaluación multicriterio.

Mihelcic, J. R., y Zimmerman, J. B. (2012). Ingeniería ambiental: Autores yEditores.

MINAM.(2011). Guíade: Diseño, construcción, operación, mantenimiento y cierre de relleno sanitario manual.

MINAM. (2011). Guía de diseño, construcción, operación, mantenimiento y cierre de relleno sanitario mecanizado.

MINAM. (2016). Plan nacional de gestión integral de residuos sólidos.

MPCH - Chachapoyas. (2009). Mejoramiento y ampliación de la gestión integral de residuos sólidos municipales de la ciudad de Chachapoyas y ampliación del servicio de disposición final de los residuos sólidos municipales de la ciudad de Huancas, provincia de Chachapoyas, departamento. Chachapoyas.

MPCH - CHACHAPOYAS. (2013). Plan de desarrollo urbano de la ciudad de Chachapoyas. Municipalid ad Provincial de Chachapoyas.

OEFA. (2015). Fiscalizacion ambiental en residuos solidos de gestión municipal, 100. Retrieved fromwebmaster@oefa.gob.pe

Paz, S. A. P. (2011). Localización de sitios adecuados para establecer un vertedero de residuos sólidos urbanos en el municipio del distrito central de Honduras.

Pucha, franz; Fries Andreas; Cánovas, Fulgencio; Oñate, Fernando; Gonzales, ctor; Pucha, D. (2017). Fundamentos de SIG.

Saralegi, B. P. (2015). Optimización de la Gestión de los Residuos Sólidos Urbanos en la Mancomunidad de San Markos mediante herramientas multicriterio.

SEMARNAT. (2002). Minimización y manejo ambiental de los residuos sólidos. Minimización y manejo ambiental de los residuos sólidos.

SIGERSOL. (2015). Caracterización de residuos sólidos de gestion municipal, distrito Chachapoyas.

Silva, L. A. (2015). Sistemas de información geográfica y la localización óptima de instalaciones para residuos sólidos: propuesta para la provincia de Huánuco.

Sosa, A. R., y Torres Romero, Crisóforo. (2010). Localización de un sitio para construir un centro de aprovechamiento de residuos sólidos urbanos a través de tres métodos. Revistas.Unam.Mx,3(1),36-48. Retrieved fromhttp://revistas.unam.mx/index.php/a idis/article/download/28656/26653

UNIDAS, N. (2015). Población, 1-5. Retrieved from http://www.un.org/es/sections/issuesdepth/population/index.html 